\title{
A Superquadratic Infeasible-Interior-Point Method for Linear Complementarity Problems
}

\author{
Stephen Wright*and Yin Zhang ${ }^{\dagger}$ \\ February 8, 1994
}

\begin{abstract}
We consider a modification of a path-following infeasible-interior-point algorithm described by Wright. In the new algorithm, we attempt to improve each new iterate by reusing the coefficient matrix factors from the latest step. We show that the modified algorithm has similar theoretical global convergence properties to the earlier algorithm, while its asymptotic convergence rate can be made superquadratic by an appropriate parameter choice.
\end{abstract}

\section{Introduction}

We describe an algorithm for solving the monotone linear complementarity problem (LCP), in which we aim to find a vector pair $(x, y)$ with

$$
y=M x+q, \quad(x, y) \geq 0, \quad x^{T} y=0
$$

where $q \in \mathbb{R}^{n}$ and $M$ is an $n \times n$ positive semidefinite matrix. The solution set to (1) is denoted by $\mathcal{S}$, while the set $\mathcal{S}^{c}$ of strictly complementary solutions is defined as

$$
\mathcal{S}^{c}=\left\{\left(x^{*}, y^{*}\right) \in \mathcal{S} \mid x^{*}+y^{*}>0\right\}
$$

Our algorithm generates positive, not neressarily feasible iterates $\left(x^{k}, y^{k}\right)$ and includes the infeasible-interior-point algorithm of Wright [10] (which is in turn based on earlier work of Zhang [12] and Wright [8]) as a special case. As in [10], the algorithm extends immediately to mixed monotone L('P' with few complications.

- Mathematics and (Computer Science Division, Argonne National Laboratory, 9700 South C'ass A venue, Argonne, Illinois 60439. The work of this author was based on research supported by the Office of Scientific. ('omputing, U.S. Department of Energy, under C'ontract W-31-109-Eng-38.

tDepartment of Mathematics and Statistics, University of Maryland Baltimore County, Baltimore, Maryland, 21228 . The work of this author was based on research supported in part by the U.S. Department of Energy under (irant DE-F(i02-93ER25171.

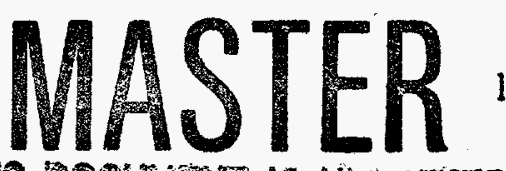


To motivate our method, we consider the following locally convergent algorithm for solving the system of nonliuear equations

$$
F(z)=0,
$$

where $F: \mathbb{R}^{N} \rightarrow \mathbb{R}^{N}$ is continuously differentiable.

Choose $\tau \in(0,1), I \geq 0, z^{0} \in \mathbb{R}^{N} ;$ Set $k \leftarrow 0$;

loop:

compute $d^{k}=-\nabla F\left(z^{k}\right)^{-1} F\left(z^{k}\right) ; z \leftarrow z^{k}+d^{k}$

for $i=0,1, \cdots, I$ (improvement loop)

compute $d=-\nabla F\left(z^{k}\right)^{-1} F(z)$

if $\quad\|F(z+d)\| \leq \tau\|F(z)\|$

then $z \leftarrow z+d$

end for

else $z^{k+1} \leftarrow z ; k \leftarrow k+1$; go to loop;

$z^{k+1} \leftarrow z ; k \leftarrow k+1$; go to loop.

On each iteration, this method takes a single Newton step and follows it up with a number of Newton-like steps calculated with the old Jacobian $\nabla F\left(z^{k}\right)$. Simple analysis shows that if $z^{*}$ is an isolated solution to the system $F(z)=0$ with $\nabla F\left(z^{*}\right)$ nonsingular, and if $\left\|z^{0}-z^{*}\right\|$ is small enough, then $\left\{z^{k}\right\}$ converges to $z^{*}$. Moreover, the inner loop (with iteration index i) eventually executes for all $I$ iterations before control passes back to the main loop and, assuming that $\nabla F(z)$ is Lipschitz continuous at $z^{*}$, the convergence has $Q$-order $I+2$ (see, for example, [6]). Note that for each value of $k$, the Jacobian $\nabla F\left(z^{k}\right)$ is evaluated and factored only once and, in many contexts, the steps $d$ calculated in the improvement loop are not expensive to compute.

Our algorithm, which we describe in Section 2, is identical to that of [10] in that it takes steps of two types - safe steps, which ensure global convergence, and fast steps, which ensure fast local convergence. As in our model algorithm above, each step is followed by an attempt to improve the new iterate without recomputing and refactoring the main coefficient matrix. The inner loop terminates when it fails to make significant progress, or after $I$ iterations, whichever comes first.

The global convergence properties of the algorithm are at least as good as the algorithm of [10] in which no attempt at improvement is made. The global convergence and complexity analysis is identical to [10]. We state the relevant results, omitting most of the details, in Section 3. In Section 4, we prove some technical results about the steps computed within the imner improvement loop, and relate them to steps computed with an exact Jacobian. Our main local convergence result is proved in Section 5 . We include some preliminary numerical results in Section 6.

In the remainder of the paper, we use $\mathbb{R}_{+}^{n}$ to denote the nonnegative orthant in $\mathbb{R}^{n}$. Subscripts on matrices and vertors indicate components, while superscripts on matrices and vectors and subscripts on scalars denote iteration numbers (usually $k$ ). 


\section{DISCLAMMER}

Portions of this document may be illegible in electronic image products. Images are produced from the best available original document. 


\section{DISCLAIMER}

This report was prepared as an account of work sponsored by an agency of the United States Government. Neither the United States Government nor any agency thereof, nor any of their employees, makes any warranty, express or implied, or assumes any legal liability or responsibility for the accuracy, completeness, or usefulness of any information, apparatus, product, or process disclosed, or represents that its use would not infringe privately owned rights. Reference herein to any specific commercial product, process, or service by trade name, trademark, manufacturer, or otherwise does not necessarily constitute or imply its endorsement, recommendation, or favoring by the United States Government or any agency thereof. The views and opinions of authors expressed herein do not necessarily state or reflect those of the United States Government or any agency thereof. 


\section{The Algorithm}

To describe the step between successive iterates, we define for any vector pair $(x, y) \in$ $\mathbb{R}_{+}^{n} \times \mathbb{R}_{+}^{n}$ the following quantities:

$$
\mu=x^{T} y / n, \quad r=y-M x-q, \quad e=(1,1, \cdots, 1)^{T},
$$

and, for any vector $x \in \mathbb{R}_{+}^{n}$,

$$
X=\operatorname{diag}(x)=\operatorname{diag}\left(x_{1}, x_{2}, \cdots, x_{n}\right) .
$$

When $(x, y)=\left(x^{k}, y^{k}\right)$ (that is, the $k$-th iterate of the algorithm), we use $r^{k}, \mu_{k}$, and $X^{k}$ to denote $r, \mu$, and $X$, respectively.

During the $k$-th iteration of the main loop, each search direction $(u, v)$ and step length $\tilde{\alpha}$ is calculated as follows.

Given $(x, y)>0, \tilde{\gamma} \in(0,1), \tilde{\beta} \in[0,1), \tilde{\sigma} \in[0,1)$, solve

$$
\left[\begin{array}{cc}
M & -I \\
Y^{k} & X^{k}
\end{array}\right]\left[\begin{array}{l}
u \\
v
\end{array}\right]=\left[\begin{array}{c}
r \\
-X Y e+\tilde{\sigma} \mu e
\end{array}\right] .
$$

Set

$$
\tilde{\alpha}=\arg \min _{\alpha \in[0, \hat{\alpha}]} \mu(\alpha) \triangleq(x+\alpha u)^{T}(y+\alpha v) / n
$$

where $\hat{\alpha}$ is the largest number in $[0,1]$ such that the following inequalities are satisfied for all $\alpha \in[0, \hat{\alpha}]$ :

$$
\begin{aligned}
(x+\alpha u)^{T}(y+\alpha v) & \geq(1-\tilde{\beta})(1-\alpha) x^{T} y, \quad \text { if } r \neq 0 \\
\left(x_{j}+\alpha u_{j}\right)\left(y_{j}+\alpha v_{j}\right) & \geq(\tilde{\gamma} / n)(x+\alpha u)^{T}(y+\alpha v), \quad j=1, \cdots, n .
\end{aligned}
$$

The inequality (4b) ensures that the componentwise products $x_{j} y_{j}$ approach zero at approximately the same rate. They stay in a loosely defined neighborhood of the central path, where $x_{j} y_{j}=\mu$ for all $j=1, \cdots, n$ - hence the term "path-following." The inequality (4a) ensures that when the current point is infeasible, the decrease in infeasibility $\|r\|$ on the current step is at least as great as the decrease in the complementarity gap $\mu$, modulo a factor of $(1-\tilde{\beta})$.

The basic form of our algorithm, given below, is the same as the one described in Wright [10], except for the addition of the improve procedure. 
Given $\bar{\gamma} \in\left(0, \frac{1}{2}\right), \gamma_{\min }$ and $\gamma_{\max }$ with $0<\gamma_{\min }<\gamma_{\max } \leq \frac{1}{2}, \ddot{\sigma} \in\left(0, \frac{1}{2}\right)$, $\rho \in(0, \bar{\gamma})$, and $\left(x^{0}, y^{0}\right)$ with $x_{j}^{0} y_{j}^{0} \geq \gamma_{\max } \mu_{0}>0$

$t_{0} \leftarrow 1, \gamma_{0} \leftarrow \gamma_{\max }, k \leftarrow 0, \nu_{0} \leftarrow 1$

while $\mu_{k}>0$

solve (2)-(4) with $(x, y)=\left(x^{k}, y^{k}\right), \tilde{\sigma}=0, \tilde{\beta}=\bar{\gamma}^{t_{k}}, \tilde{\gamma}=\gamma_{\min }+\bar{\gamma}^{t_{k}}\left(\gamma_{\max }-\gamma_{\min }\right)$

if $\quad\left(x^{k}+\tilde{\alpha} u\right)^{T}\left(y^{k}+\tilde{\alpha} v\right) / n \leq \rho \mu_{k}$

then $\beta_{k_{k}} \leftarrow \tilde{\beta}, t \leftarrow t_{k}+1, \gamma \leftarrow \tilde{\gamma}$

else solve (2)-(4) with $(x, y)=\left(x^{k}, y^{k}\right), \tilde{\sigma} \in\left[\bar{\sigma}, \frac{1}{2}\right], \tilde{\beta}=0, \tilde{\gamma}=\gamma_{k}$;

end if

$\beta_{k} \leftarrow 0, t \leftarrow t_{k}, \gamma \leftarrow \gamma_{k} ;$

$\alpha_{k} \leftarrow \tilde{\alpha}, \sigma_{k} \leftarrow \tilde{\sigma}, y \leftarrow \nu_{k}\left(1-\alpha_{k}\right),(x, y) \leftarrow\left(x^{k}, y^{k}\right)+\alpha_{k}(u, v) ;$

improve $\left((x, y), t, u, \gamma,\left(x^{k}, y^{k}\right)\right)$;

end while.

$$
t_{k+1} \leftarrow t, \nu_{k+1} \leftarrow \nu, \gamma_{k+1} \leftarrow \gamma,\left(x^{k+1}, y^{k+1}\right) \leftarrow(x, y), k \leftarrow k+1 ;
$$

We refer to the steps that are computed with $\tilde{\sigma}=0$ as fast steps, because they lead to rapid local convergence, while the steps with $\tilde{\sigma} \in\left[\bar{\sigma}, \frac{1}{2}\right]$ are safe steps, because they ensure global convergence.

The improve procedure, which reuses the coefficient matrix in (2) to improve the new iterate, takes a combination of safe and fast steps, just like the main algorithm. The main difference is that the procedure is terminated if an improvement in $\mu$ of at least a factor of $\tau \in(\rho, 1)$ is not achieved. The user supplies the parameter $\tau$ and the nonnegative integer $I$, where $I$ is the maximum number of steps that can be taken in improve.

improve $\left((x, y), t, \nu, \gamma,\left(x^{k}, y^{k}\right)\right)$

Given $\tau \in(\rho, 1), I \geq 0$,

for $i=1,2, \cdots, I$

if $\quad \mu=0$ then return;

solve (2)-(4) with $\tilde{\sigma}=0, \tilde{\beta}=\bar{\gamma}^{t}, \tilde{\gamma}=\gamma_{\text {min }}+\bar{\gamma}^{t}\left(\gamma_{\max }-\gamma_{\text {min }}\right)$;

if $\quad(x+\tilde{\alpha} u)^{T}(y+\tilde{\alpha} v) / n \leq \rho \mu$

then $t \leftarrow t+1, \gamma \leftarrow \tilde{\gamma}$;

else solve (2)-(4) with $\tilde{\sigma} \in\left[\bar{\sigma}, \frac{1}{2}\right], \tilde{\beta}=0, \tilde{\gamma}=\gamma$;

if $\quad(x+\tilde{\alpha} u)^{T}(y+\tilde{\alpha} v) / n>\tau \mu$ then return;

end if

end for.

$\nu \leftarrow \nu(1-\tilde{\alpha}),(x, y) \leftarrow(x, y)+\tilde{\alpha}(u, v) ;$

In the special case $I=0$, improve is vacuous and the algorithm reduces to the method 
of [10]. We refer the reader to that paper for the intuitive motivation behind the use of safe and fast steps.

The inclusion of improve does not alter some of the fundamental properties of the iteration sequeuce $\left(x^{k}, y^{k}\right)$. We still have

$$
r^{k}=\nu_{k} r^{0}
$$

and also the following result, which is similar to Lemma 3.1 of [9].

Lemma 2.1 Suppose that the initial point is infeasible, that is, $r^{0} \neq 0$. Then the positive constant $\hat{\beta}$ defined by

$$
\hat{\beta}=\prod_{k=1}^{\infty}\left(1-\bar{\gamma}^{k}\right)
$$

is such that

$$
\mu_{k} \geq \hat{\beta} \nu_{k} \mu_{0}=\hat{\beta} \frac{\left\|r^{k}\right\|}{\left\|r^{0}\right\|} \mu_{0}, \quad \forall k \geq 0 .
$$

We also have the following result, which shows that the algorithm either terminates finitely at a solution of (1) or else generates an infinite sequence $\left\{\left(x^{k}, y^{k}\right)\right\}$ of strictly positive iterates. The proof is a simple modification of [10, Lemma 3.2] and is omitted.

Lemma 2.2 For all iterates generated by the algorithm, we have either $\left(x^{k}, y^{k}\right)>0$ or else $\mu_{k}=0$.

We assume throughout the remainder of the paper that finite termination does not occur, that is, all iterates $\left(x^{k}, y^{k}\right)$ and all the intermediate points $(x, y)$ generated in the improve procedure are strictly positive.

\section{Global Convergence}

The analysis of global convergence and polynomial complexity is nearly identical to that of $\left[10\right.$, Section :3]. We need only note that (5) still applies and that all iterates $\left(x^{k}, y^{k}\right)$ satisfy $x_{j}^{k} y_{j}^{k} \geq \gamma_{\text {min }} \mu_{k}, j=1, \cdots, n$. The intermediate points generated by improve have the same properties. The technical results from [10, Section 3] can therefore be applied to show that nontrivial progress is made at each safe step. The presence of improve and the fast steps cannot hinder (and very often speed) the convergence.

In this section we summarize the main results from [10, Section 3] and state the sole assumption required for global convergence, which is as follows.

Assumption $1 \mathcal{S} \neq \emptyset$.

Theorem 3.1 If a safe step is taken at itcration $k$, then there is a constant $\omega>0$ such that the step length $\alpha_{k}$ has

$$
\alpha_{k} \geq \frac{1}{\omega}
$$


If the initial point $\left(x^{0}, y^{0}\right)$ is chosen as

$$
\left(x^{0}, y^{0}\right)=\left(\xi_{x} e, \xi_{y} e\right)
$$

where

$$
\xi_{x} \geq\left\|x^{*}\right\|_{\infty}, \quad \xi_{y} \geq\left\|y^{*}\right\|_{\infty}, \quad \xi_{y} \geq\|q\|_{\infty}, \quad \xi_{y} \geq\|M e\|_{\infty} \xi_{x}=\left\|M x^{0}\right\|_{\infty},
$$

for some $\left(x^{*}, y^{*}\right) \in \mathcal{S}$, then $\omega=O\left(n^{2}\right)$.

Proof. See [10, Lemma 3.4, Theorem 3.5], where a different definition of $\omega$ is used.

The main global convergence result is as follows.

Theorem 3.2 The complementarity gap $\mu_{k}$ converges geometrically to zero.

Proof. As in Wright [10, Theorem 3.6], we can show that if a safe step is taken at iteration $k$, we have

$$
\left(x^{k}+\alpha_{k} u\right)^{T}\left(y^{k}+\alpha_{k} v\right) / n \leq\left(1-\frac{1}{4 \omega}\right) \mu_{k},
$$

while if a fast step is taken, we have

$$
\left(x^{k}+\alpha_{k} u\right)^{T}\left(y^{k}+\alpha_{k} v\right) / n \leq \rho \mu_{k} .
$$

Since the complementarity gap may be decreased further by improve, we have $\mu_{k+1} \leq$ $\left(x^{k}+\alpha_{k} u\right)^{T}\left(y^{k}+\alpha_{k} v\right) / n$ and therefore

$$
\mu_{k+1} \leq \max \left(1-\frac{1}{4 \omega}, \rho\right) \mu_{k}
$$

from which the result follows.

Finally, we state the polynomial complexity result.

Theorem 3.3 [10, Corollary 3.7] Let $\epsilon>0$ be given. Suppose that the starting point is defined by (6). (7), where $\mu_{0}=\xi_{x} \xi_{y} \leq 1 / \epsilon^{\tau}$ for some constant $\tau \geq 0$ independent of $n$. Then there is an integer $K_{\text {e }}$ with

$$
K_{c}=O\left(n^{2} \log (1 / \epsilon)\right)
$$

such that $\mu_{k} \leq \epsilon$ for all $k \geq K_{\text {. }}$.

\section{Technical Results}

In the remainder of the paper, we turn our attention to the latter stages of the algorithm. We show that the algorithm eventually takes only fast steps (that is, the then branch of the main conditional statement is executed). Moreover, the improve routine eventually takes fast steps on all $I$ of its iterations, so that a total of $I+1$ fast steps are taken for each factorization of the coefficient matrix in (2). 
In this section, we prove some results about the steps generated in this fast phase of the algorithm. In particular, we look at the effects of the inexact coefficient matrix in (2) on the steps calculated within improve.

We start by defining the two assumptions for the local convergence analysis, which will be implicitly assumed to hold throughout the remainder of the paper.

Assumption $2 \mathcal{S}^{c} \neq \emptyset$.

Assumption $3 \mathcal{S}$ is bounded.

For monotone LCP, a sufficient condition for Assumption 3 is the existence of a strictly feasible pair $(\bar{x}, \bar{y})$ such that $\bar{y}=M \bar{x}+q,(\bar{x}, \bar{y})>0$. This can be seen from the fact that for any $\left(x^{*}, y^{*}\right) \in \mathcal{S}$

$$
\left(x^{*}-\bar{x}\right)^{T}\left(y^{*}-\bar{y}\right)=\left(x^{*}-\bar{x}\right)^{T} M\left(x^{*}-\bar{x}\right) \geq 0,
$$

implying

$$
\bar{x}^{T} y^{*}+\bar{y}^{T} x^{*} \leq \bar{x}^{T} \bar{y}
$$

By choosing any particular strictly complementary solution $\left(x^{*}, y^{*}\right)$, we can define index sets $B$ and $N$ by

$$
B=\left\{j \mid x_{j}^{*}>0\right\}, \quad N=\left\{j \mid y_{j}^{*}>0\right\} .
$$

It is well known that the global convergence of the algorithm guarantees that the iteration sequence $\left\{\left(x^{k}, y^{k}\right)\right\}$ approaches the solution set $\mathcal{S}$ (see the error bound result of Mangasarian [2], for example). Therefore, Assumption 3 implies the boundedness of the iteration sequence $\left\{\left(x^{k}, y^{k}\right)\right\}$, as given in the following lemma.

Lemma 4.1 There is a positive constant $C_{3}$ such that $\left\|\left(x^{k}, y^{k}\right)\right\| \leq C_{3}$ for all $k \geq 0$.

The next two results are simple modifications of results from Wright [10, Section 4]. Since we will apply these results to intermediate points generated by improve as well as to the main iterates $\left(x^{k}, y^{k}\right)$, we state them in a more general form than in [10]. The proofs are, however, not affected. Boundedness of the iteration sequence is not necessary for either result, and neither is Assumption 3.

Lemma 4.2 ([10, Lemma 4.1]) Let $(x, y) \geq 0$ be such that

$$
r=y-M x-q=v r^{\circ} \quad \text { for some } \nu \in\left[0, \frac{1}{2}\right] \text {, }
$$

and $\mu=x^{T} y / n \geq \hat{B} \nu \mu_{0}$ for this value of $\nu$. Then for some constant $C_{4}>0$ we have

$$
\left\|x_{N}\right\| \leq C_{4} \mu, \quad\left\|y_{B}\right\| \leq C_{4} \mu \text {. }
$$

Lemma 4.3 Let $(x, y)$ be any point with the properties defined in Lemma 4.2, and suppose in addition that $x_{j} y_{j} \geq \gamma_{\min } \mu$. Let $(\vec{u}, \bar{v})$ be the scarch dirction obtained by solving

$$
\left[\begin{array}{cc}
M & -I \\
Y & X
\end{array}\right]\left[\begin{array}{c}
\bar{u} \\
\bar{v}
\end{array}\right]=\left[\begin{array}{c}
r \\
-X Y e+\tilde{\sigma} \mu e
\end{array}\right],
$$


where $\tilde{\sigma} \in[0,1)$. Then there exists a positive constant $C_{5}$ such that

$$
\left\|\bar{u}_{N}\right\| \leq C_{5} \mu, \quad\left\|\bar{v}_{B}\right\| \leq C_{5} \mu .
$$

If in addition $\tilde{\sigma}=0$, there is a constant $C_{6}>0$ such that

$$
\left\|\bar{u}_{B}\right\| \leq C_{6} \mu, \quad\left\|\bar{v}_{N}\right\| \leq C_{6} \mu .
$$

Proof. Follows from Lemma 4.2 and Theorem 4.5 of [10].

We now turu to the "approximate" fast steps computed by $(2)$, where $(x, y)$ is either the current iterate $\left(x^{k}, y^{k}\right)$ or some intermediate point generated in the call to improve at iteration $k$. It is obvious from the algorithm definition that we have

$$
\mu=x^{T} y / n \leq \mu_{k} .
$$

We also assume that the point $(x, y)$ is not too far from $\left(x^{k}, y^{k}\right)$ in the sense that there is a constant $\chi \geq 1$ independent of $k$ such that

$$
\left\|\left(x^{k}-x, y^{k}-y\right)\right\| \leq \chi \mu_{k} .
$$

The following result describes some characteristics of the actual search direction $(u, v)$ calculated from (2), partly in terms of the exact search direction $(\bar{u}, \bar{v})$ that satisfies $(9)$.

Lemma 4.4 Let $(x, y)$ be a vector pair satisfying the assumptions of Lemma 4.3 and, in addition, the properties (12) and (13). Then if $\tilde{\sigma}=0$, there are positive constants $C_{7}, C_{8}$, and $C_{9}$ independent of $k$ and $\chi$ such that the following bounds are satisfied:

$$
\begin{gathered}
\|u-\bar{u}\| \leq C_{7} \chi \mu, \quad\|v-\bar{v}\| \leq C_{7} \chi \mu, \\
\|(u, v)\| \leq C_{8} \chi \mu, \\
\left\|\bar{u}_{N}-u_{N}\right\| \leq C_{9} \chi \mu \mu_{k}, \quad\left\|\bar{v}_{B}-v_{B}\right\| \leq C_{9} \chi \mu \mu_{k} .
\end{gathered}
$$

Froof. From (2), we have that

$$
\left[\begin{array}{cc}
M & -I \\
Y^{k} & X^{k}
\end{array}\right]\left[\begin{array}{l}
u \\
v
\end{array}\right]=\left[\begin{array}{c}
r \\
-X Y e
\end{array}\right]
$$

while from (9), we have

$$
\left[\begin{array}{cc}
M & -I \\
Y & X
\end{array}\right]\left[\begin{array}{l}
\bar{u} \\
\bar{v}
\end{array}\right]=\left[\begin{array}{c}
r \\
-X Y e
\end{array}\right]
$$

and therefore

$$
\left[\begin{array}{cc}
M & -I \\
Y^{k} & X^{k}
\end{array}\right]\left[\begin{array}{c}
\bar{u} \\
\bar{v}
\end{array}\right]=\left[\begin{array}{c}
r \\
-X Y e+\left(Y^{k}-Y\right) \bar{u}+\left(X^{k}-X\right) \bar{v}
\end{array}\right]
$$


From (17) and (18) we obtain

$$
\left[\begin{array}{cc}
M & -I \\
Y^{k} & X^{k}
\end{array}\right]\left[\begin{array}{c}
\bar{u}-u \\
\bar{v}-v
\end{array}\right]=\left[\begin{array}{c}
0 \\
\left(Y^{k}-Y\right) \bar{u}+\left(X^{k}-X\right) \bar{v}
\end{array}\right] .
$$

Now from (1:3) and Lemma 4.3, there is a constant $\bar{C}_{7}$ independent of $k$ and $\chi$ such that

$$
\left\|\left(Y^{k}-Y\right) \bar{u}+\left(X^{k}-X\right) \bar{v}\right\| \leq \bar{C}_{7} \times \mu \mu_{k} .
$$

Defining

$$
D^{k}=\left(X^{k}\right)^{-1 / 2}\left(Y^{k}\right)^{1 / 2}
$$

and multiplying the lower block in the system (19) by $\left(X^{k} Y^{k}\right)^{-1 / 2}$, we obtain

$$
D^{k}(\bar{u}-u)+\left(D^{k}\right)^{-1}(\bar{v}-v)=\left(X^{k} Y^{k}\right)^{-1 / 2}\left[\left(Y^{k}-Y\right) \bar{u}+\left(X^{k}-X\right) \bar{v}\right] .
$$

Using the upper block of (19), we have $(\bar{v}-v)=M(\bar{u}-u)$, and so it follows from positive semidefiniteness of $M$ that

$$
(\bar{u}-u)^{T}(\bar{v}-v) \geq 0 .
$$

By taking the Euclidean norm of both sides of (21), and using (22), we have

$$
\left\|D^{k}(\bar{u}-u)\right\|^{2}+\left\|\left(D^{k}\right)^{-1}(\bar{v}-v)\right\|^{2} \leq\left\|\left(X^{k} Y^{k}\right)^{-1 / 2}\right\|^{2}\left\|\left(Y^{k}-Y\right) \bar{u}+\left(X^{k}-X\right) \bar{v}\right\|^{2}
$$

Therefore

$$
\begin{aligned}
\left\|D^{k}(\bar{u}-u)\right\| & \leq\left\|\left(X^{k} Y^{k}\right)^{-1 / 2}\right\|\left\|\left(Y^{k}-Y\right) \bar{u}+\left(X^{k}-X\right) \bar{v}\right\| \\
\left\|\left(D^{k}\right)^{-1}(\bar{v}-v)\right\| & \leq\left\|\left(X^{k} Y^{k}\right)^{-1 / 2}\right\|\left\|\left(Y^{k}-Y\right) \bar{u}+\left(X^{k}-X\right) \bar{v}\right\|
\end{aligned}
$$

Now since $x_{j}^{k} y_{j}^{k} \geq \gamma_{\text {min }} \mu_{k}$, we have

$$
\left\|\left(X^{k} Y^{k}\right)^{-1 / 2}\right\|=\max _{j=1, \cdots, n}\left(x_{j}^{k} y_{j}^{k}\right)^{-1 / 2} \leq \gamma_{\min }^{-1 / 2} \mu_{k}^{-1 / 2} .
$$

Therefore from (20) we have

$$
\left\|D^{k}(\bar{u}-u)\right\| \leq \bar{C}_{\tau} \times \gamma_{\text {unu }}^{-1 / 2} \mu \mu_{k}^{1 / 2}
$$

Taking any $j=1, \cdots, n$, we find that

$$
\left|\frac{\left(y_{j}^{k}\right)^{1 / 2}}{\left(x_{j}^{k}\right)^{1 / 2}}\left(\bar{u}_{j}-u_{j}\right)\right| \leq\left\|D^{k}(\bar{u}-u)\right\| \leq \bar{C}_{7} \chi \gamma_{\min }^{-1 / 2} \mu \mu_{k}^{1 / 2} .
$$


Hence,

$$
\begin{aligned}
\left|\bar{u}_{j}-u_{j}\right| & \leq \max _{j=1, \cdots, n} \frac{\left(x_{j}^{k}\right)^{1 / 2}}{\left(y_{j}^{k}\right)^{1 / 2}} \bar{C}_{7} \chi \gamma_{\text {min }}^{-1 / 2} \mu \mu_{k}^{1 / 2} \\
& \leq \max _{j=1, \cdots, n} \frac{x_{j}^{k}}{\left(x_{j}^{k} y_{j}^{k}\right)^{1 / 2}} \bar{C}_{7} \chi \gamma_{\min }^{-1 / 2} \mu \mu_{k}^{1 / 2} \\
& \leq \frac{C_{3}}{\gamma_{\min }^{1 / 2} \mu_{k}{ }_{k}^{1 / 2}} \bar{C}_{7} \chi \gamma_{\min }^{-1 / 2} \mu \mu_{k}^{1 / 2} \\
& \leq \frac{C_{7}}{\sqrt{n}} \chi \mu,
\end{aligned}
$$

for $C_{7}$ defined in an obvious way. We have proved the first inequality in (14); the proof of the second inequality is similar.

For (16), we repeat the logic above to obtain for $i \in N$ that

$$
\begin{aligned}
\left|\bar{u}_{j}-u_{j}\right| & \leq \frac{\left(x_{j}^{k}\right)^{1 / 2}}{\left(y_{j}^{k}\right)^{1 / 2}} \bar{C}_{7} \chi \gamma_{\min }^{-1 / 2} \mu \mu_{k}^{1 / 2} \\
& \leq \frac{x_{j}^{k}}{\left(x_{j}^{k} y_{j}^{k}\right)^{1 / 2}} \bar{C}_{7} \chi \gamma_{\min }^{-1 / 2} \mu \mu_{k}^{1 / 2} \\
& \leq \frac{C_{4} \mu_{k}}{\gamma_{\text {nin }}^{1 / 2} \mu_{k}^{1 / 2}} \bar{C}_{7} \chi \gamma_{\min }^{-1 / 2} \mu \mu_{k}^{1 / 2} \\
& \leq \frac{C_{9}}{\sqrt{n}} \chi \mu \mu_{k},
\end{aligned}
$$

where $C_{9}$ is defined appropriately. The bound for $\left\|\bar{v}_{B}-v_{B}\right\|$ follows similarly.

To prove (15), we have from Lemma 4.3 and (14) that

$$
\|(u, v)\| \leq\|(\bar{u}, \bar{v})\|+\|(u-\bar{u}, v-\bar{v})\| \leq 2\left(C_{5}+C_{6}\right) \mu+2 C_{7} \chi \mu \leq C_{8} \chi \mu,
$$

where we have defined $C_{8}=2\left(C_{5}+C_{6}+C_{7}\right)$ and used the assumption that $\chi \geq 1$.

We now state the main result of this section, in which we obtain an estimate for the step length $\tilde{\alpha}$ along a (possibly approximate) fast step direction $(u, v)$. The point $(x, y)$ considered in this theorem represents either the main iterate $\left(x^{k}, y^{k}\right)$ itself or one of the intermediate points generated by improve during iteration $k$. For the purpose of this result, we define the following positive constants, all of which are independent of $k$ and $\chi$ :

$$
\begin{aligned}
& \bar{C}_{10}=C_{9}\left(C_{3}+C_{6}\right)+C_{7}\left(C_{4}+C_{5}\right) \\
& C_{10}=2\left(C_{5} C_{6}+\bar{C}_{10}+C_{7} C_{9}\right) \\
& C_{12}=\frac{2 C_{10}}{(1-\bar{\gamma})\left(\gamma_{\max }-\gamma_{\min }\right)} \\
& C_{13}=2\left(C_{3} C_{9}+C_{4} C_{7}+C_{8}^{2}\right), \\
& C_{14}=C_{12}+C_{13} / n .
\end{aligned}
$$


Theorem 4.5 Let $(x, y)$ be a point that satisfies the assumptions of Lemma 4.4, and in addition

$$
\mu \leq \min \left(1, \frac{n}{C_{13} \chi^{2}}\right) .
$$

Let $t$ be a positive integer such that for $\gamma$ defined by

$$
\gamma=\gamma_{\min }+\bar{\gamma}^{t-1}\left(\gamma_{\max }-\gamma_{\min }\right)
$$

we have $x_{j} y_{j} \geq \gamma \mu$ for $j=1, \cdots, n$, and suppose for this value of $t$ that

$$
C_{14} \chi^{2} \frac{\mu_{k}}{\bar{\gamma}^{t}} \leq \rho .
$$

Then if a fast step is attempted from the point $(x, y)$ with

$$
\tilde{\sigma}=0, \quad \tilde{\beta}=\bar{\gamma}^{t}, \quad \tilde{\gamma}=\gamma_{\text {min }}+\bar{\gamma}^{t}\left(\gamma_{\max }-\gamma_{\text {min }}\right),
$$

and the search direction $(u, v)$ is calculated from (Q), the resulting step length $\tilde{\alpha}$ obtained from (2), (3), and (4) satisfics

$$
\tilde{\alpha} \geq 1-C_{12} \chi^{2} \frac{\mu_{k}}{\bar{\gamma}^{t}} .
$$

Moreover, the fast step is accepted with

$$
(x+\tilde{\alpha} u)^{T}(y+\tilde{\alpha} v) / n \leq C_{14} \chi^{2} \frac{\mu_{k}}{\bar{\gamma}^{t}} \mu .
$$

Proof. The proof is in three stages. First, we show that the tests (4) are satisfied for all $\alpha$ in the range

$$
\left[0,1-C_{12} \chi^{2} \frac{\mu_{k}}{\bar{\gamma}^{t}}\right] \text {. }
$$

Second, we show that $\mu(\alpha)$ defined by (3) is decreasing on the interval $\alpha \in[0,1]$. In the third stage, we show that

$$
\mu(\tilde{\alpha}) \leq C_{14} \chi^{2} \frac{\mu_{k}}{\bar{\gamma}^{t}} \mu \leq \rho \mu,
$$

which proves the result.

We first consider the condition (4a). From the left-hand side, we obtain

$$
\begin{aligned}
& (x+\alpha u)^{T}(y+\alpha v) \\
& =(x+\alpha \bar{u}+\alpha(u-\bar{u}))^{T}(y+\alpha \bar{v}+\alpha(v-\bar{v})) \\
& =x^{T} y(1-\alpha)+\alpha^{2} \bar{u}^{T} \bar{v}+\alpha(x+\alpha \bar{u})^{T}(v-\bar{v})+\alpha(y+\alpha \bar{v})^{T}(u-\bar{u}) \\
& \quad+\alpha^{2}(u-\bar{u})^{T}(v-\bar{v}) .
\end{aligned}
$$

Now, using Lemma 4.1 and the inequalities (8), (10), (11), (14), (15), and (16), we have

$$
\left|\bar{u}^{T} \bar{v}\right| \leq 2 C_{5} C_{6} \mu^{2} \leq 2 C_{5} C_{66} \mu \mu_{k},
$$


and

$$
\begin{aligned}
\left|(u-\bar{u})^{T}(y+\alpha \bar{v})\right| & \leq\left\|u_{N}-\bar{u}_{N}\right\|\left(\left\|y_{N}\right\|+\left\|\bar{v}_{N}\right\|\right)+\left\|u_{B}-\bar{u}_{B}\right\|\left(\left\|y_{B}\right\|+\left\|\bar{v}_{B}\right\|\right) \\
& \leq C_{9} \chi \mu \mu_{k}\left(C_{3}+C_{6} \mu\right)+C_{7} \chi \mu^{2}\left(C_{4}+C_{5}\right) \\
& \leq \bar{C}_{10} \chi \mu \mu_{k},
\end{aligned}
$$

where we have used $\mu \leq 1$ and $\mu \leq \mu_{k}$ to derive the last inequality. Similarly, we have

$$
\left|(v-\bar{v})^{T}(x+\alpha \bar{u})\right| \leq \bar{C}_{10} \times \mu \mu_{k}
$$

while for the remaining term in (28) we have from Lemma 4.4 that

$$
\left|(u-\bar{u})^{T}(v-\bar{v})\right| \leq 2 C_{7} C_{9} \chi^{2} \mu^{2} \mu_{k} \leq 2 C_{7} C_{9} \chi^{2} \mu \mu_{k}
$$

Hence, since $\chi \geq 1$, we have from the definition of $C_{10}$ that

$$
\left|(x+\alpha u)^{T}(y+\alpha v)-(1-\alpha) x^{T} y\right| \leq C_{10} \chi^{2} \mu \mu_{k}
$$

Since $\tilde{\beta}=\bar{\gamma}^{t}$, we bave that $(4 a)$ is satisfied provided that

$$
\left(\gamma_{10} \chi^{2} \mu \mu_{k} \leq(1-\alpha) \bar{\gamma}^{t} n \mu\right.
$$

which is certainly true provided that

$$
1-\alpha \geq \frac{C_{10} \chi^{2} \mu_{k}}{n \bar{\gamma}^{t}}
$$

From the definition of $C_{12}$, since $1-\bar{\gamma}$ and $\gamma_{\max }-\gamma_{\text {mun }}$ both lie in the range $(0,1)$, we have

$$
\frac{C_{10} \chi^{2} \mu_{k}}{n \bar{\gamma}^{t}} \leq \frac{C_{12} \chi^{2} \mu_{k}}{\bar{\gamma}^{t}}
$$

so the inequality (4a) certainly holds for all $\alpha$ in the range (26).

Turning to the second inequality (4b), we have by an argument similar to the one above. that

$$
\left(x_{j}+\alpha u_{j}\right)\left(y_{j}+\alpha v_{j}\right) \geq x_{j} y_{j}(1-\alpha)-C_{10} \chi^{2} \mu \mu_{k} \geq \gamma \mu(1-\alpha)-C_{10} \chi^{2} \mu \mu_{k},
$$

while from (29), we have

$$
(x+\alpha u)^{T}(y+\alpha v) / n \leq(1-\alpha) \mu+C_{10} \chi^{2} \mu \mu_{k} / n
$$

Hence, the inequality $(4 b)$ holds provided that

$$
\tilde{\gamma} \mu(1-\alpha)+C_{10} \chi^{2} \mu \mu_{k}(\tilde{\gamma} / n) \leq \gamma \mu(1-\alpha)-C_{10} \chi^{2} \mu \mu_{k},
$$

which is certainly true whenever the inequality

$$
(\gamma-\tilde{\gamma})(1-\alpha) \geq 2 C_{10} \chi^{2} \mu_{\kappa}
$$


holds. Since

$$
\gamma-\dot{\gamma}=\left[\gamma_{\min }+\bar{\gamma}^{t-1}\left(\gamma_{\max }-\gamma_{\min }\right)\right]-\left[\gamma_{\text {min }}+\bar{\gamma}^{t}\left(\gamma_{\max }-\gamma_{\min }\right)\right]=\bar{\gamma}^{t-1}(1-\bar{\gamma})\left(\gamma_{\max }-\gamma_{\min }\right),
$$

we find that (30) holds whenever

$$
1-\alpha \geq \frac{2 C_{10} \chi^{2} \mu_{k}}{\bar{\gamma}^{t-1}(1-\bar{\gamma})\left(\gamma_{\max }-\gamma_{\min }\right)},
$$

which, by definition of $C_{12}$, is true for $\alpha$ in the range (26).

For the second part of the proof, we show that $\mu(\alpha)$ defined by (3) is decreasing on the range $\alpha \in[0,1]$. Taking the derivative, we have

$$
\begin{aligned}
n \mu^{\prime}(\alpha) & =\left(x^{T} v+y^{T} u\right)+2 \alpha u^{T} v \\
& =\left(x^{T} \bar{v}+y^{T} \bar{u}\right)+x^{T}(v-\bar{v})+y^{T}(u-\bar{u})+2 \alpha u^{T} v \\
& =-x^{T} y+x^{T}(v-\bar{v})+y^{T}(u-\bar{u})+2 \alpha u^{T} v .
\end{aligned}
$$

However, we can use Lemma 4.1 and relations (8), (14), and (16) to obtain

$$
\begin{aligned}
\left|x^{T}(v-\bar{v})\right| & \leq\left\|x_{B}\right\|\left\|v_{B}-\bar{v}_{B}\right\|+\left\|x_{N}\right\|\left\|v_{N}-\bar{v}_{N}\right\| \\
& \leq C_{3} C_{9} \chi \mu \mu_{k}+C_{4} C_{7} \chi \mu^{2} \\
& \leq\left(C_{3} C_{9}+C_{4} C_{7}\right) \chi \mu \mu_{k},
\end{aligned}
$$

where we have used $\mu \leq \mu_{k}$ in the last inequality. A similar bound can be obtained for $\left|y^{T}(u-\bar{u})\right|$. For the final term in (31), we have

$$
\left|u^{T} v\right| \leq C_{8}^{2} \chi^{2} \mu^{2} \leq C_{8}^{2} \chi^{2} \mu \mu_{k} .
$$

Substituting these relations in (31) and using the definition of $C_{13}$, we obtain

$$
n \mu^{\prime}(\alpha) \leq\left[-n+C_{13} \chi^{2} \mu_{k}\right] \mu .
$$

It follows from (2:3) that the term in brackets is negative and hence $\mu^{\prime}(\alpha) \leq 0$ for all $\alpha \in[0,1]$.

Finally, we observe that the step length $\tilde{\alpha}$ actually selected by the procedure will be at least as long as the upper bound of (26), so using (32), (33), and the definitions of $C_{13}$ and $C_{14}$, we have

$$
\begin{aligned}
(x & +\tilde{\alpha} u)^{T}(y+\tilde{\alpha} v) \\
& \leq x^{T} y(1-\tilde{\alpha})+\left|x^{T}(v-\bar{v})\right|+\left|y^{T}(u-\bar{u})\right|+\left|u^{T} v\right| \\
& \leq\left(x^{T} y\right) C_{12} \chi^{2} \frac{\mu_{k}}{\bar{\gamma}^{t}}+2\left(C_{3} C_{9}+C_{4} C_{7}\right) \chi \mu \mu_{k}+C_{8}^{2} \chi^{2} \mu \mu_{k} \\
& \leq\left(x^{T} y\right) \frac{\mu_{k}}{\bar{\gamma}^{t}}\left[C_{12}+C_{13} / n\right] \chi^{2} \\
& =\left(x^{T} y\right) \frac{\mu_{k}}{\bar{\gamma}^{t}} C_{14} \chi^{2} .
\end{aligned}
$$


Therefore (25) holds. Acceptance of this step follows from (24), since we have $(x+\tilde{\alpha} u)^{T}(y+$ $\tilde{\alpha} v) / n \leq \rho \mu$.

We close this section with a result that is important in defining the onset of the algorithm's fast phase.

Lemma 4.6 There is a constant $\eta<1$ such that

$$
\frac{\mu_{k+1}}{\bar{\gamma}^{t_{k+1}}} \leq \eta \frac{\mu_{k}}{\bar{\gamma}^{t_{k}}}, \quad \forall k \geq 0 .
$$

Proof. When the safe branch of the main algorithm is taken at iteration $k$, we have from the proof of Theorem 3.2 that

$$
\left(x^{k}+\tilde{\alpha} u\right)^{T}\left(y^{k}+\tilde{\alpha} v\right) / n \leq\left(1-\frac{1}{4 \omega}\right) \mu_{k},
$$

while the value of $t$ is unaltered. It is possible that in the subsequent call to improve, the value of $t$ will be incremented. Whenever this happens, we are guaranteed that the complementarity gap $\mu$ decreases by a factor of at least $\rho$, so the ratio $\mu / \bar{\gamma}^{t}$ will also decrease by a factor of at least $\rho / \bar{\gamma}<1$. Hence, when the safe branch is taken, we have

$$
\frac{\mu_{k+1}}{\bar{\gamma}^{t_{k+1}}} \leq\left(1-\frac{1}{4 \omega}\right) \frac{\mu_{k}}{\bar{\gamma}^{t_{k}}} .
$$

When the fast branch is taken, we have $t \leftarrow t+1$ and

$$
\left(x^{k}+\tilde{\alpha} u\right)^{T}\left(y^{k}+\tilde{\alpha} v\right) / n \leq \rho \mu_{k},
$$

so the ratio $\mu / \bar{\gamma}^{t}$ decreases by a factor of at least $\rho / \bar{\gamma}$. The comments above ensure that the subsequent rall to improve can only accentuate this decrease, so in this case we have

$$
\frac{\mu_{k+1}}{\bar{\gamma}^{t_{k+1}}} \leq \frac{\rho}{\bar{\gamma}} \frac{\mu_{k}}{\bar{\gamma}^{t_{k}}} .
$$

The result is obtained by defining

$$
\eta=\max \left(1-\frac{1}{4 \omega}, \frac{\rho}{\bar{\gamma}}\right) .
$$

\section{Local Convergence}

In this section, we state and prove our two main local convergence results. First, we define a threshold value of $\mu_{k} / \bar{\gamma}^{t_{k}}$ below which both the main algorithm and the procedure improve take unly fast steps. Second, we sbow that the resulting superlinear convergence has Q-order $I+2$. 
Theorem 5.1 Define

$$
\chi=2\left(C_{5}+C_{6}\right) \exp \left(\frac{C_{8} \rho}{1-\rho}\right),
$$

and let $K_{1}$ be the smallest inde $x$ such that $\nu_{K_{1}} \leq 1 / 2$,

$$
C_{14} \chi^{2} \frac{\mu_{K_{1}}}{\bar{\gamma}^{t_{K_{1}}+1}} \leq \rho
$$

and

$$
\mu_{K_{1}} \leq \min \left(1, \frac{n}{C_{13} \chi^{2}}\right)
$$

Then the fast branch is taken in the main algorithm and, moreover, I fast steps are taken in the call to improve.

Proof. Existence of $K_{1}$ is guaranteed by Lemma 4.6. We choose any $k \geq K_{1}$. Our proof proceeds by showing first that the step taken from $\left(x^{k}, y^{k}\right)$ in the main algorithm is a fast step. We then prove by induction that $I$ fast steps are taken inside the procedure improve. Our main tool in both cases is Theorem 4.5.

For the first part of the proof, we apply Theorem 4.5 with

$$
(x, y)=\left(x^{k}, y^{k}\right), \quad t=t_{k}, \quad \gamma=\gamma_{k} .
$$

Note that the point $(x, y)$ satisfies the assumptions of Lemmas 4.2 and 4.3 (by definition of $K_{1}, r^{k}, \nu_{k}$, etc.) and the conditions (12) and (13) (trivially). Clearly also $x_{j}^{k} y_{j}^{k} \geq \gamma_{k} \mu_{k}$ for all $j=1, \cdots, n$, and the condition (2:3) also holds. Because

$$
C_{14} \chi^{2} \frac{\mu_{k}}{\bar{\gamma}^{l}}=C_{14} \chi^{2} \frac{\mu_{k}}{\bar{\gamma}^{t_{k}}} \leq C_{14} \chi^{2} \frac{\mu_{K_{1}}}{\bar{\gamma}^{t_{K_{1}}}} \leq C_{14} \chi^{2} \frac{\mu_{K_{1}}}{\bar{\gamma}^{t_{K_{3}}+l}} \leq \rho,
$$

the condition (24) also bolds. Hence the conditions of Theorem 4.5 are satisfied by the choices (38), and therefore a fast step is taken by the main algorithm.

We turn now to the procedure improve. Our aim is to show inductively that if $(x, y)$ is the current vector pair at the commencement of the $i$-th iteration of this procedure, then

$$
\left\|\left(x^{k}, y^{k}\right)-(x, y)\right\| \leq\left[2\left(C_{5}+C_{6}\right) \prod_{l=1}^{i-1}\left(1+C_{8} \rho^{l}\right)\right] \mu_{k} .
$$

Moreover, we show that a fast step is taken from this vector $(x, y)$ during the $i$-th iteration of improve. Note for future reference that

$$
\log \prod_{l=1}^{i-1}\left(1+C_{8} \rho^{l}\right)=\sum_{l=1}^{i-1} \log \left(1+C_{8} \rho^{l}\right) \leq \sum_{l=1}^{i-1} C_{8} \rho^{l} \leq \frac{C_{8} \rho}{1-\rho},
$$

and so

$$
2\left(C_{5}+C_{63}\right) \prod_{l=1}^{i-1}\left(1+C_{8} \rho^{l}\right) \leq \chi, \quad i=1, \cdots, l
$$


Consider the case $i=1$, that is, the first iteration of improve. We aim to use Theorem 4.5 again, so we start by checking that the point just generated by the main algorithm satisfies the assumptions of this theorem. In other words, the choices

$$
(x, y)=\left(x^{k}, y^{k}\right)+\alpha_{k}(u, v), \quad t=t_{k}+1,
$$

must be shown to satisfy these assumptions. It is easy to see that the assumptions of Lemmas 4.2 and 4.3 and the condition (12) are satisfied. To see (13), note that the fast step just taken at iteration $k$ of the main algorithm was computed with an exact coefficient matrix, that is, we have $(\bar{u}, \bar{v})=(u, v)$. Hence we can apply Lemma 4.3 to deduce that

$$
\left\|\left(x^{k}, y^{k}\right)-(x, y)\right\| \leq\|(u, v)\|=\|(\bar{u}, \bar{v})\| \leq 2\left(C_{5}+C_{6}\right) \mu_{k} .
$$

Thus the bound (39), and therefore also (13), holds for this point $(x, y)$. The conditions (23) and $x_{j} y_{j} \geq \gamma \mu$ clearly hold, while (24) also holds because

$$
C_{14} \chi^{2} \frac{\mu_{k}}{\bar{\gamma}^{\ell}}=C_{14} \chi^{2} \frac{\mu_{k}}{\bar{\gamma}^{t_{k}+1}} \leq C_{14} \chi^{2} \frac{\mu_{k}}{\bar{\gamma}^{t_{k}+l}} \leq C_{14} \chi^{2} \frac{\mu_{K_{1}}}{\bar{\gamma}^{t_{1}}+I} \leq \rho .
$$

Hence Theorem 4.5) applies, and we have shown that a fast step is taken on the first iteration of improve.

We now consider the general iteration $i$ of the internal loop of improve. We assume that our assertions hold for iterations 1 through $i-1$. Let $\left(x^{-}, y^{-}\right)$denote the value of $(x, y)$ at the start of iteration $i-1$, and let $\left(u^{-}, v^{-}\right)$be the search direction calculated during this iteration, while as before $(x, y)$ is the current point at the start of iteration $i$. To obtain an estimate of $\left\|\left(x^{k}, y^{k}\right)-(x, y)\right\|$, we note by our inductive hypothesis (39) that

$$
\left\|\left(x^{k}, y^{k}\right)-\left(x^{-}, y^{-}\right)\right\| \leq\left[2\left(C_{5}+C_{6}\right) \prod_{l=1}^{i-2}\left(1+C_{8} \rho^{l}\right)\right] \mu_{k} .
$$

We now apply Lemma 4.4 to the step $\left(u^{-}, v^{-}\right)$taken during iteration $i-1$, with $\chi$ replaced by $2\left(C_{5}+C_{6}\right) \prod_{l=1}^{i-2}\left(1+C_{8} \rho^{l}\right)$, to find that

$$
\begin{aligned}
& \left\|\left(x^{k}, y^{k}\right)-(x, y)\right\| \\
& \quad \leq\left\|\left(x^{k}, y^{k}\right)-\left(x^{-}, y^{-}\right)\right\|+\left\|\left(x^{-}, y^{-}\right)-(x, y)\right\| \\
& \quad \leq 2\left(C_{5}+C_{6}\right) \prod_{l=1}^{i-2}\left(1+C_{8} \rho^{l}\right) \mu_{k}+\left\|\left(u^{-}, v^{-}\right)\right\| \\
& \quad \leq 2\left(C_{5}+C_{6}\right) \prod_{l=1}^{i-2}\left(1+C_{8} \rho^{l}\right) \mu_{k}+C_{8}\left[2\left(C_{5}+C_{6}\right) \prod_{l=1}^{i-2}\left(1+C_{8} \rho^{l}\right)\right] \mu^{-} \\
& \quad \leq\left[2\left(C_{5}+C_{6}\right) \prod_{l=1}^{i-2}\left(1+C_{8} \rho^{l}\right)\right]\left(1+C_{8} \rho^{i-1}\right) \mu_{k} .
\end{aligned}
$$

The final inequality follows from the fart that $\mu^{-} \leq \rho^{i-1} \mu_{k}$, since $\left(x^{-}, y^{-}\right)$is arrived at by taking $i-1$ fast steps (one step in the main algorithm, followed by $i-2$ iterations of the 
improve loop), at each of which a reduction factor of at least $\rho$ is achieved. We have now shown that the bound (39) continues to hold at iteration $i$. It is easy to check that the remaining conditions required by Theorem 4.5 hold. We mention only (24), which holds for $t=t_{k}+i$ because

$$
C_{14} \chi^{2} \frac{\mu_{k}}{\bar{\gamma}^{t}}=C_{14} \chi^{2} \frac{\mu_{k}}{\bar{\gamma}^{t_{k}+i}} \leq C_{14} \chi^{2} \frac{\mu_{k}}{\bar{\gamma}^{t_{k}+1}} \leq C_{14} \chi^{2} \frac{\mu_{K_{1}}}{\bar{\gamma}^{t_{1}+1}} \leq \rho .
$$

Hence, we can apply Theorem 4.5 again to deduce that a fast step is taken at iteration $i$, and our result is proved.

Our final result is to show high-order convergence of the sequence $\left\{\mu_{k}\right\}$ to zero. We show that this convergence has a $Q$-order of at least $I+2$, that is, for any $\epsilon>0$

$$
\limsup _{k \rightarrow \infty} \frac{\mu_{k+1}}{\mu_{k}^{1+2-\epsilon}}=0 .
$$

An equivalent characterization of the $Q$-order $I+2$ convergence is the inequality (40) below (see. Potra [7]).

Theorem 5.2 The subsequence $\left\{\mu_{k}\right\}, k=0,1, \cdots$, converges to zero with $Q$-order $I+2$, that is,

$$
\liminf _{k \rightarrow \infty} \frac{\log \mu_{k+1}}{\log \mu_{k}} \geq I+2
$$

Proof. Consider $k \geq K_{1}$. Since a fast step is taken by the main algorithm and all $I$ iterations of improve, and since Theorem 4.5 applies at all $I+1$ steps, we can apply the inequality (25) $I+1$ times to bound $\mu_{k+1}$ in terms of $\mu_{k}$. The process yields

$$
\mu_{k+1} \leq C_{14}^{l+1} \chi^{2(I+1)} \frac{\mu_{k}^{l+2}}{\bar{\gamma}^{(I+1) t_{k}+I(I+1) / 2}} .
$$

It follows from Lemma 4.6 and (41) that

$$
\frac{\mu_{k+1}}{\mu_{k}} \leq \frac{C_{14}^{I+1} \chi^{2(I+1)}}{\bar{\gamma}^{I(I+1) / 2}}\left(\frac{\mu_{k}}{\bar{\gamma}^{t_{k}}}\right)^{I+1} \rightarrow 0
$$

that is, $\left\{\mu_{k}\right\}$ converges to zero at least $Q$-superlinearly.

By taking logarithms, we obtain from (41) that

$$
\log \mu_{k+1} \leq \log \left(\frac{C_{14}^{I+1} \chi^{2(I+1)}}{\bar{\gamma}^{I(I+1) / 2}}\right)+(I+2) \log \mu_{k}-(I+1) t_{k} \log \bar{\gamma}
$$

We will assume that $k$ is sufficiently large such that $\mu_{k}<1$. From the above,

$$
\frac{\log \mu_{k+1}}{\log \mu_{k}} \geq I+2+\log \left(\frac{C_{14}^{I+1} \chi^{2(I+1)}}{\bar{\gamma}^{I(I+1) / 2}}\right) / \log \mu_{k}-(I+1) \log \bar{\gamma} \frac{t_{k}}{\log \mu_{k}} .
$$


Obviously, as $k \rightarrow \infty$, the second term in the right-hand side vanishes. If we can show that the third term also goes to zero, then the conclusion (40) follows. Since $t_{k} \leq(I+1) k+1$, it suffices to prove

$$
\lim _{k \rightarrow \infty} \frac{k}{\log \mu_{k}}=0 .
$$

Suppose otherwise. Then there exist $\xi \in(0,1)$ and a subsequence $\left\{\mu_{k}\right\}_{\mathcal{K}} \subset\left\{\mu_{k}\right\}$ such that for all $k \in K$ :

$$
\frac{k}{\log \mu_{k}} \leq \frac{1}{\log \xi}, \text { or equivalently } \xi^{k} \leq \mu_{k} .
$$

From (42), there exists a positive integer $J$ sucb that for all $k \geq J, \mu_{k+1} \leq \frac{\xi}{2} \mu_{k}$. Hence, for all $k>J$ and $k \in X$,

$$
\xi^{k} \leq \mu_{k} \leq\left(\frac{\xi}{2}\right)^{k-J} \mu_{J} .
$$

That is, for all $k>J$ and $k \in k:, 2^{k} \leq \mu_{J} 2^{J} / \xi^{J}$. This is clearly a contradiction.

\section{$6 \quad$ Numerical Examples}

We include some preliminary numerical results that compare the bebavior of our algorithm with the method of [10], in which improve is vacuous $(I=0)$.

Our test problems have $M=A \Lambda A^{T}$, where $A \in \mathbb{R}^{n \times n}$ is dense with elements drawn from a uniform distribution in $[-1,1]$, and $\Lambda$ is a diagonal matrix with diagonal elements $\Lambda_{i i}=10^{4 \zeta_{i}}$, where $\zeta_{i}$ is drawn from a uniform distribution in $[0,1]$. A solution $\left(x^{*}, y^{*}\right)$ is generated so that even-numbered components of $x^{*}$ and odd-numbered components of $y^{*}$ are zero, and $q$ is chosen so that the nonzero components of both vectors are uniformly distributed in $[0,1]$.

The algorithmic constants have the following values:

$$
\begin{aligned}
\gamma_{\min }=10^{-6}, \quad \gamma_{\max } & =.002, \quad \sigma_{\min }=10^{-3}, \quad \sigma_{\max }=.1, \\
\bar{\gamma} & =.25, \quad \rho=.99 \bar{\gamma} .
\end{aligned}
$$

We also modify the algorithms slightly so that only safe steps are attempted when the current value of $\mu$ is greater than 1 (that is, the fast step branch of the conditional statements in both the main algorithm and improve is bypassed). The value of $\tilde{\sigma}$ for the safe step at iteration $k$ is chosen as

$$
\sigma_{k}=\operatorname{mid}\left(\sigma_{\text {min }}, \mu_{k} / \sqrt{n}, \sigma_{\max }\right),
$$

where mid() denotes the median of its three arguments. Termination occurs when $\mu_{k} \leq$ $10^{-10}$.

Performance of the algorithm for $\tau=.8$ and $\tau=.9$ is shown in Tables 1 and 2 , respectively. We tabulate the number of factorizations (which equals the number of iterations of the algorithm), together with the tutal number of linear system solutions performed, and the 
total number of corrector steps taken in improve. The behavior on these random problems is not too seusitive to the choices of the parameters $I$ and $\tau$; the choices $I=3$ and $\tau=.8$ would probably be good choices in general.

Table 1: Performance of the algorithm for $\tau=.8$

\begin{tabular}{|c|c|c|c|c|}
\hline & & $n=10$ & $n=50$ & $n=100$ \\
\hline \multirow{3}{*}{$I=0$} & factorizations & 24 & 38 & 38 \\
\hline & solves & 36 & 56 & 54 \\
\hline & corrector steps & 0 & 0 & 0 \\
\hline \multirow{3}{*}{$I=1$} & factorizations & 20 & 33 & 34 \\
\hline & solves & 59 & 99 & 97 \\
\hline & corrector steps & 10 & 11 & 10 \\
\hline \multirow{3}{*}{$I=2$} & factorizations & 18 & 31 & 32 \\
\hline & solves & 65 & 110 & 105 \\
\hline & corrector steps & 14 & 17 & 15 \\
\hline \multirow{3}{*}{$I=3$} & factorizations & 18 & 31 & 31 \\
\hline & solves & 72 & 112 & 109 \\
\hline & corrector steps & 17 & 20 & 19 \\
\hline \multirow{3}{*}{$I=5$} & factorizations & 17 & 30 & 31 \\
\hline & solves & 74 & 114 & 113 \\
\hline & corrector steps & 22 & 23 & 23 \\
\hline
\end{tabular}

Table 2: Performance of the algorithm for $\tau=.9$

\begin{tabular}{|ll|ccc|}
\hline & & $n=10$ & $n=50$ & $n^{\prime}=100$ \\
\hline \multirow{2}{*}{$I=1$} & factorizations & 20 & 33 & 33 \\
& solves & 59 & 99 & 93 \\
& corrector steps & 10 & 11 & 12 \\
\hline \multirow{2}{*}{$I=2$} & factorizations & 18 & 31 & 32 \\
& solves & 65 & 110 & 105 \\
& corrector steps & 14 & 17 & 15 \\
\hline \multirow{2}{*}{$I=3$} & factorizations & 17 & 31 & 31 \\
& solves & 74 & 114 & 109 \\
& corrector steps & 20 & 21 & 19 \\
\hline \multirow{3}{*}{$I=5$} & factorizations & 17 & 30 & 31 \\
& solves & 76 & 114 & 115 \\
& corrector steps & 23 & 24 & 24 \\
\hline
\end{tabular}




\section{Final Comments}

In this paper, we analyze an infeasible-interior-point algorithm that reuses matrix factors to accelerate convergence. In addition to the usual global convergence properties, the new algorithm possesses a local convergence rate of $Q$-order $I+2$.

The idea of reusing matrix factors was utilized in a number of works on interior-point methods. Among them, Mehrotra [3] and Zhang and Zhang [11] are most closely related to the current work. Mehrotra [3] also obtained a $Q$-order of $I+2$ convergence result, but it is for a feasible-interior-point algorithm. Moreover, his algorithm is in the Mizuno-ToddYe [5] predictor-torrector framework, thus always requiring two matrix factorizations per iteration. Zhang and Zhang [11] analyzed an infeasible-interior-point algorithm with $I=1$ that asymptotically requires only one matrix factorization per iteration. However, they only obtained $Q$-order 2 convergence instead of $Q$-order 3.

The higher-order convergence rates are probably of theoretic interest only. In practice, it is difficult to observe on computer a convergence rate higher than cubic. As can be seen from our preliminary numerical results, however, the approach of reusing matrix factors does have the tendency to reduce the number of factorizations required for solving LCP problems at a price of increasing the number of back solves. Since at each iteration matrix factorization is the dominant work in comparison to back solves, the potential reduction in computational work could be significant for large-scale LCP problems. For linear programming, the practical effectiveness of reusing matrix factors is already well documented $[1,4]$.

\section{References}

[1] I. J. Lustic, R. E. Marsten, and D. F. Shanno, On implementing Mehrotra's predictor-corrector interior point method for linear programming, SIAM Journal of Optimization, 2 (1992), pp. 435-449.

[2] O. L. MANGiASARIAN, Error bounds for nondegenerate monotone linear complementarity problems, Mathematical Programming, 48 (1990), pp. 437-445.

[3] S. MEHROTRA, Asymptotic convergence in a generalized predictor-corrector method, Technical Report, Dept. of Industrial Engineering and Management Science, Northwestern University, Evanston, Ill., October 1992.

[4] - On the implementation of a primal-dual interior point method, SIAM Journal of Optimization, 2 (1992), pp. 575-601.

[5] S. Mizuno, M. TODD, AND Y. YE, On adaptive step primal-dual interior-point algorithms for lincar programming, Mathematics of Operations Research, 18 (1993), pp. 964981 .

[6] J. M. ORTEGA AND W. C. RHEINBOLDT, Iterative solution of nonlinear equations in scveral variables, Acarlemic Press, New York and London, 1970. 
[7] F. A. Potra, On Q-order and R-order of convergence, Journal of Optimization Theory and Applications, 63 (1989), pp. 415-431.

[8] S. J. WRIGHT, An infeasible-interior-point algorithm for linear complementarity problems, Preprint MCS-P331-1092, Mathematics and Computer Science Division, Argonne National Laboratory, Argonne, Ill., October 1992. Mathematical Programming, to appear.

[9] —_, A path-following infeasible-interior-point algorithm for linear complementarity problems, Optimization Methods and Software, 2 (1993), pp. 79-106.

[10] — A path-following interior-point algorithm for linear and quadratic optimization problems, Preprint MCS-P401-1293, Mathematics and Computer Science Division, Argonne National Laboratory, Argonne, Ill., December 1993.

[11] D. ZHANG AND Y. ZHANG, A Mehrotra-type predictor-corrector algorithm with polynomial complexity and Q-subquadratic convergence, Tech. Rep. 93-13, Department of Mathematics and Statistics, University of Maryland Baltimore County, September 1993.

[12] Y. ZHANc; On the convergence of a class of infeasible-interior-point methods for the horizontal linear complementarity problem, Tech. Rep. 92-07, Department of Mathematics and Statistics, University of Maryland Baltimore County, April 1992. SIAM J. Optimization, to appear. 